\title{
PREVALÊNCIA DE SÍNDROME METABÓLICA EM PACIENTES COM ESQUIZOFRENIA REFRATÁRIA ${ }^{1}$
}

\author{
METABOLIC SYNDROME PREVALENCE IN PATIENTS WITH \\ REFRACTORY SCHIZOPHRENIA
}

\section{PREVALENCIA DE SÍNDROME METABÓLICO EN PACIENTES CON ESQUIZOFRENIA REFRACTARIA}

\author{
Pedro Henrique Batista de Freitas ${ }^{*}$ \\ Paulo Afonso Granjeiro ** \\ Bianca Penido Vecchia ${ }^{* * *}$ \\ Mayara Lopes de Paula ${ }^{* * * *}$ \\ Mayra Cristina TaVares ${ }^{* * * * *}$ \\ RichaRdSON Miranda MACHADO ${ }^{* * * * *}$
}

\begin{abstract}
RESUMO
Objetivo: Estimar prevalência de síndrome metabólica (SM) e seus fatores associados em pacientes com esquizofrenia refratária em uso do antipsicótico clozapina. Método: Trata-se de um estudo descritivo e transversal, realizado na Região Ampliada Oeste do Estado de Minas Gerais (MG), em 2015, com uma amostra de 72 pacientes. Foram coletados dados sociodemográficos, clínicos, antropométricos e bioquímicos. Realizou-se análise descritiva, univariada e multivariada. Resultados: Observou-se prevalência de SM em 47,2\% da amostra, com predomínio entre as mulheres $(58,8 \%)$. Pacientes com SM apresentaram percentuais mais elevados de alterações, principalmente em relação à glicemia e triglicérides. O uso de quatro ou mais medicamentos e a presença de sobrepeso e obesidade estiveram associados à SM. Além disso, pacientes com a síndrome apresentaram um histórico de menos internações psiquiátricas, comparados àqueles que não a possui. Conclusão: $\mathrm{A}$ prevalência de SM encontrada nos pacientes com esquizofrenia refratária foi elevada e alarmante. A presença de sobrepeso e obesidade e o uso de 4 ou mais medicamentos podem estar associados com o desenvolvimento de SM neste grupo. Essa taxa pode representar um importante indicador de risco cardiovascular, sendo sugerida a construção de estratégias de prevenção primária das alterações metabólicas, bem como se indica que o paciente seja acompanhado periodicamente, principalmente em relação aos componentes da SM.
\end{abstract}

Palavras chave: Esquizofrenia, Síndrome X Metabólica, clozapina, enfermagem cardiovascular.

${ }^{1}$ Artigo extraído da dissertação de mestrado intitulada: Esquizofrenia refratária: prevalência de síndrome metabólica e qualidade de vida de pacientes em uso de clozapina. Programa de Pós-Graduação em Enfermagem da Universidade Federal de São João Del-Rei (UFSJ), Divinópolis, Minas Gerais, Brasil, 2015.

* Enfermeiro. Mestre em Enfermagem pela Universidade Federal de São João Del-Rei (UFSJ). Divinópolis, Minas Gerais, Brasil. E-mail: pedrohbf@yahoo.com.br

${ }^{* *}$ Bioquímico. Doutor em Bioquímica pela UNICAMP. Professor adjunto da UFSJ. Divinópolis, Minas Gerais, Brasil. E-mail: pagranjeiro@gmail.com

${ }^{* * *}$ Acadêmica de Enfermagem pela UFSJ. Divinópolis, Minas Gerais, Brasil. E-mail: biancavecchia@hotmail.com

${ }^{* * * *}$ Acadêmica de Enfermagem pela UFSJ. Divinópolis, Minas Gerais, Brasil. E-mail: mayara.lopesp@hotmail.com

${ }^{* * * * *}$ Acadêmica de Enfermagem pela UFSJ. Divinópolis, Minas Gerais, Brasil. E-mail: mayracristinatavares@hotmail.com

${ }^{* * * * * *}$ Enfermeiro. Doutor em Psiquiatria pela USP. Professor adjunto da UFSJ. Divinópolis, Minas Gerais, Brasil. E-mail: richardson@usp.br 


\section{ABSTRACT}

Objective: To estimate the prevalence of the metabolic syndrome (MS) and its associated factors in patients with refractory schizophrenia using the antipsychotic clozapine. Method: This is a descriptive cross-sectional study conducted in the extended western region of Minas Gerais (MG), Brazil, in 2015, using a sample of 72 patients. We collected sociodemographic, clinical, anthropometric and biochemical data and conducted descriptive univariate and multivariate analysis. Results: We verified the prevalence of MS in $47.2 \%$ of the sample, with a greater predominance among women (58.8\%). Patients with MS showed higher change values, especially in relation to blood glucose and triglycerides. The use of four or more medications and the presence of overweight and obesity were associated with MS. In addition, patients with the syndrome had fewer cases of psychiatric hospitalizations than those who did not have it. Conclusion: High and alarming levels of MS prevalence were found in patients with refractory schizophrenia. The presence of overweight and obesity and the use of 4 or more medications may be associated with the development of the MS in this group. These levels could represent an important indicator of cardiovascular risk, which raises the need to develop strategies for primary prevention of metabolic alterations, and highlights the importance of a periodical monitoring of the patient, especially regarding the components of the MS.

Key words: Schizophrenia. Metabolic Syndrome X, clozapine, cardiovascular nursing.

\section{RESUMEN}

Objetivo: Estimar la prevalencia del síndrome metabólico (SM) y sus factores asociados en pacientes con esquizofrenia refractaria que utilizan clozapina como antipsicótico. Material y método: Se trata de un estudio descriptivo y transversal realizado en la región ampliada del oeste de Minas Gerais (MG) en 2015, con una muestra de 72 pacientes. Se recogieron datos sociodemográficos, clínicos, antropométricos y bioquímicos. Se realizó análisis descriptivo, univariado y multivariado. Resultados: Se observó prevalencia de SM en el 47,2\% de la muestra, con una prevalencia entre las mujeres (58,8\%). Los pacientes con SM tenían mayores porcentajes de alteración, especialmente en la glucosa en sangre y triglicéridos. El uso de cuatro o más medicamentos y la presencia de sobrepeso y obesidad se asociaron con SM. Además, los pacientes con el síndrome tenían un historico de hospitalizaciones psiquiátricas menor que los que no lo tienen. Conclusión: La prevalencia del SM encontrado en pacientes con esquizofrenia refractaria fue alto y alarmante. La presencia de sobrepeso y obesidad, y el uso de 4 o más fármacos puede estar asociado con el desarrollo de la SM en este grupo. Esta tasa puede representar un importante indicador de riesgo cardiovascular, y sugiere la construcción de estrategias de prevención primaria de los cambios metabólicos, así como que el paciente debe controlarse periódicamente, especialmente en relación con los componentes del SM.

Palabras clave: Esquizofrenia, Síndrome X Metabólico, clozapina, enfermería cardiovascular.

Fecha recepción: 04/01/16 Fecha aceptación: 05/09/16

\section{INTRODUÇÃO}

A esquizofrenia é um transtorno mental complexo e incapacitante, que afeta mais de 21 milhões de pessoas em todo o mundo. É caracterizada por um conjunto de sinais e sintomas que incluem discurso e comportamento desorganizados, delírios, alucinações e alteração na cognição (1). O tratamento baseia-se essencialmente no uso de medicamentos denominados antipsicóticos, associados a terapias psicossociais (2).

Cerca de $30 \%$ das pessoas que possuem 
esquizofrenia não apresentam melhora do quadro clínico, sendo denominadas refratárias ao tratamento (3). Apesar de não existir um consenso único e globalmente aceito, a esquizofrenia refratária pode ser caracterizada, segundo consenso atual, quando não há melhora dos principais sintomas após o tratamento com dois antipsicóticos de classes diferentes (sendo pelo menos um atípico), em doses adequadas, durante um determinado período de tempo $(3,4)$. O conceito original de esquizofrenia refratária foi proposto por Kane em 1995 e determinou os seguintes critérios diagnósticos: resposta parcial, durante pelo menos cinco anos, a três diferentes tipos de antipsicóticos (pelos menos dois com estruturas químicas diferentes); intolerância aos efeitos adversos; recaídas ou deterioração sintomática, mesmo utilizando-se doses apropriadas dos medicamentos (5). Outro conceito mundialmente aceito como critério de esquizofrenia refratária inclui a duração do transtorno por mais de cinco anos e sintomas produtivos que não melhoram após o uso regular e na dosagem máxima com pelo menos dois tipos de antipsicóticos (6).

O tratamento medicamentoso de escolha para os casos de esquizofrenia refratária é o uso do antipsicótico atípico clozapina. A clozapina é um antipsicótico de segunda geração que acarreta importante melhora clínica dos pacientes e, consequentemente, de sua qualidade de vida $(1,3)$. Todavia, seu uso não está isento de efeitos adversos, podendo provocar, principalmente, alterações metabólicas graves e persistentes, como ganho de peso, aumento da adiposidade central, dislipidemia, intolerância à glicose e resistência à insulina, que são relacionados ao desenvolvimento da síndrome metabólica (SM) (7) .

A SM caracteriza-se por um conjunto de fatores de risco para diabetes mellitus e doenças cardiovasculares, tendo como componentes centrais a obesidade abdominal, resistência à insulina, dislipidemia e hipertensão. Além disso, também está associada ao desenvolvimento de doenças hepáticas e câncer
(8). Em pacientes com esquizofrenia, estimase elevado percentual de mortes por doença cardiovascular, acometendo, aproximadamente, 34\% nos homens e 31\% nas mulheres (9). Desta forma, apresenta-se como uma condição complexa que acarreta alto custo social e econômico para os sistemas de saúde e famílias, atingindo todas as populações do mundo (2).

A etiologia da SM ainda não está bem elucidada, mas a adiposidade central e a resistência à insulina parecem desempenhar importante papel na sua ocorrência (8). O critério diagnóstico mais utilizado é o proposto pelo The National Cholesterol Education Program (NCEP) adapted Adult Treatment Panel III (ATP IIIA), que define essa condição quando da presença de três ou mais fatores clínicos de cinco avaliados estão alterados, sendo eles: aumento da pressão arterial, glicemia, triglicérides e circunferência abdominal, além de baixos níveis de HDL colesterol (10).

A prevalência da SM depende da definição adotada e de seus componentes, bem como da composição da população em estudo (sexo, idade, raça e etnia). Estima-se que entre 10 a $30 \%$ da população mundial tenha SM, sendo mais prevalente nas mulheres e em pessoas com mais idade (11). Pessoas que possuem esquizofrenia apresentam um risco de desenvolver SM duas vezes maior, em comparação ao da população geral. Fatores de risco como etnia, início tardio e longa duração da doença, tabagismo e uso de antipsicóticos contribuem para o seu desenvolvimento (12). Apesar da relação causal entre antipsicóticos e SM não estar completamente esclarecida, existe evidência significativa da associação entre os medicamentos de segunda-geração e o desenvolvimento dessa síndrome, principalmente no que tange à clozapina, que é utilizada pela maioria dos pacientes que possuem esquizofrenia refratária (13).

A prevalência de SM em pessoas com esquizofrenia também depende da definição e metodologia utilizada, além de características da população em estudo, podendo variar 
entre 11 e 69\%, embora uma recente meta-análise ter estimado taxa de aproximadamente $32,5 \%(14,15)$. Estudos epidemiológicos relacionados à esquizofrenia refratária são escassos, não sendo encontradas pesquisas no Brasil e em países da América Latina envolvendo esta temática. Existem evidências de que pessoas com esquizofrenia refratária que utilizam clozapina estão expostas a um risco mais elevado para SM em comparação à esquizofrenia de modo geral, podendo alcançar o dobro, representando um grave problema de saúde pública a nível mundial (16).

Nesta perspectiva observa-se que a SM em pessoas com esquizofrenia, principalmente naquelas que possuem a forma refratária, pode representar um importante e valioso indicador de risco para condições que impactam significativamente na morbidade e mortalidade desse grupo. Por conseguinte, conhecer a prevalência e os possíveis fatores associados torna-se ferramenta essencial e útil para o planejamento de políticas e estratégias voltadas à sua prevenção, tratamento e controle.

Este artigo foi elaborado com o objetivo de estimar prevalência de síndrome metabólica e seus fatores associados em pacientes com esquizofrenia refratária em uso do antipsicótico clozapina.

\section{MATERIAL E MÉTODO}

Trata-se de um estudo transversal e analítico, realizado na Região Ampliada de Saúde Oeste de Minas Gerais, com pacientes com diagnostico de esquizofrenia refratária em uso do antipsicótico clozapina. Foram respeitados os seguintes critérios de inclusão: diagnostico médico de esquizofrenia refrataria em uso do antipsicótico atípico clozapina, maiores de 18 anos, de ambos os sexos e com capacidade de entendimento. Foram excluídos do estudo as mulheres grávidas, os participantes que não estavam em jejum e aqueles que apresentaram qualquer condição que pudesse interferir na coleta e na mensuração dos dados, como, por exemplo, a presença deficiência física que pudesse prejudicar a aferição das características antropométricas.

O cálculo amostral foi realizado utilizando-se o programa OpenEpi versão 3.03a, considerando-se uma população de 169 indivíduos para uma proporção esperada do evento de $50 \%$, um nível de significância de $5 \%$ e margem de erro de $10 \%$, estimando-se uma amostra de aproximadamente 62 indivíduos. A amostra final foi constituída por 72 participantes. Desse total, 51 (70\%) faziam uso de clozapina há mais de 5 anos. $\mathrm{O}$ projeto foi aprovado pelo Comitê de Ética em Pesquisa com Seres Humanos da Universidade Federal de São João Del-Rei, Campus Centro-Oeste Dona Lindu (UFSJ/CCO) sob o parecer 572.288.

O período da coleta de dados compreendeu os meses de dezembro de 2014 a junho de 2015. Os pacientes com esquizofrenia refratária da Região Ampliada de Saúde Oeste de Minas Gerais foram previamente convidados para a coleta de dados por meio do envio de cartas e da realização de contato telefônico, quando receberam todas as orientações necessárias à realização da pesquisa. Os dados foram coletados no Centro de Atenção Psicossocial tipo III do município-polo da Região Ampliada Oeste, de acordo com a data e o horário indicado.

Para a coleta de dados sociodemográficos, clínicos e comportamentais foi utilizado um questionário semi-estruturado, précodificado e padronizado. As entrevistas e a coleta de dados antropométricos e bioquímicos foram realizadas em salas devidamente preparadas. Para mensurar o HDLcolesterol, os níveis de triglicérides e glicose, foram retiradas amostras de sangue venoso da veia cubital do antebraço, após jejum de $12 \mathrm{~h}$, e a análise foi feita no laboratório de bioquímica da Universidade Federal de São João Del-Rei/ Campus Centro-Oeste Dona Lindu (UFSJ/CCO), utilizando o analisador 
automático BS120 Bioclin.

A verificação do peso e da altura foi realizada seguindo-se técnicas padronizadas. Para mensurar o peso foi utilizada balança eletrônica digital marca Filizola ${ }^{\circledR}$ (Indústria Filizola S/A, Brasil), com precisão de $0,1 \mathrm{~kg}$ e capacidade de $150 \mathrm{~kg}$. O paciente foi posicionado de pé, no centro da base da balança, descalço e com roupas leves. Em relação à altura foi utilizado o estadiômetro com haste móvel da balança marca Filizola ${ }^{\circledR}$ (São Paulo, Brasil), modelo 31 , com precisão de $0,5 \mathrm{~cm}$. A circunferência da cintura (CC) foi obtida com o participante em posição ereta, ao final da expiração, no ponto médio entre o último arco costal e a crista ilíaca ântero-superior, utilizando-se de fita métrica inelástica e flexível, com precisão de $0,1 \mathrm{~cm}$.

A mensuração da pressão arterial (PA) seguiu todos os critérios e passos preconizados na VI Diretrizes Brasileiras de Hipertensão Arterial (17). A medida da PA foi realizada pelo método indireto com técnica auscultatória, utilizando-se de esfigmomanômetro do tipo aneroide devidamente calibrado. $\mathrm{O}$ procedimento foi realizado duas vezes com intervalos de dois minutos entre cada medida, sendo o valor final determinado pela média das medidas.

$\mathrm{O}$ índice de massa corporal (IMC) foi calculado usando-se a fórmula "IMC = peso $(\mathrm{kg}) /$ altura $^{2}(\mathrm{~m})$ " e classificado de acordo com os pontos de corte estabelecidos pela WHO (18). A SM foi definida utilizandose dos critérios do The National Cholesterol Education Program (NCEP) adapted Adult Treatment Panel III (ATP IIIA), quando da presença de três ou mais dos seguintes fatores de risco: obesidade central (circunferência abdominal $>102 \mathrm{~cm}$ em homens ou $>88$ $\mathrm{cm}$ em mulheres); pressão arterial elevada $(>130 / 85 \mathrm{~mm} \mathrm{Hg}$ ) ou em tratamento com anti-hipertensivo; hiperglicemia (glicemia em jejum $>100 \mathrm{mg} \mathrm{dL}$ ) ou em tratamento com hipoglicemiante; concentração elevada de triglicérides (>150 $\mathrm{mg} \mathrm{dL}$ ) ou em uso de medicação para reduzi-lo; HDL colesterol baixo ( $<40 \mathrm{mg}$ dL em homens ou $<50 \mathrm{mg} \mathrm{dL}$ em mulheres) ou em uso de medicação para HDL baixo (19).

O processamento e a análise dos dados foram realizados por meio do programa Statistical Package for the Social Science ${ }^{\circledR}$, versão 20.0. As variáveis foram descritas por meio de suas frequências absolutas e relativas. Para a comparação dos pacientes com e sem síndrome metabólica foram utilizados os testes de Qui-Quadrado de Pearson e exato de Fisher. Na análise multivariada, para avaliar os fatores associados à SM, foi utilizado o modelo de regressão logística binária. Para a entrada das variáveis preditoras no modelo, foi considerado um valo-p menor que 0,20 na análise univariada. Após ajuste do modelo final, foi avaliada a estimativa do Odds Ratio ajustado com o respectivo intervalo de confiança de 95\% (IC 95\%). O ajuste do modelo foi avaliado por meio da estatística de Hosmer \& Lemeshow.

\section{RESULTADOS}

Neste estudo foram avaliados 72 pacientes com esquizofrenia refratária, em uso de clozapina, sendo encontrada, não intencionalmente, a mesma proporção para ambos os sexos (50\%). A média de idade foi de 42,9 anos e a maioria dos participantes eram solteiros $(72,2 \%)$.

A Tabela 1 apresenta a prevalência de $\mathrm{SM}$ e a distribuição de seus componentes alterados. Observa-se que a prevalência foi de $47,2 \%$, sendo mais predominante nas mulheres $(58,8 \%)$, em comparação aos homens $(41,2 \%)$. Quanto à distribuição de seus componentes, nota-se que a hiperglicemia $(66,7 \%)$ e a hipertrigliceridemia $(63,9 \%)$ foram os que apresentaram os maiores percentuais.

Todas as variáveis analisadas na Tabela 2 mostraram associação significativa com a ocorrência de SM (valores-p $<0,05)$. Nes- 
ta investigação, o aumento do IMC está associado maior prevalência de SM. Entre os participantes sem a SM, a prevalência de obesidade foi de $21,6 \%$, enquanto que entre aqueles com a síndrome esse percentual aumenta para 38,2\%. Em relação ao sobrepeso, a prevalência em pacientes com a síndrome foi de $47,1 \%$, enquanto naqueles sem a SM essa taxa caiu para $24,3 \%$.

Os resultados apresentados na Tabela 3 apontam que as variáveis sociodemográficas não se relacionaram com a SM em pessoas com esquizofrenia refratária nesta investigação. As variáveis que apresentaram associação significativa com a ocorrência de SM foram "internado em hospital psiquiátrico", "número de medicamentos" e "comorbidades". Por outro lado, os pacientes com SM tomam 4 ou mais medicamentos mais frequentemente $(80,6 \%)$ que aqueles sem a síndrome $(50 \%)$. Além disso, os participantes com SM apresentaram maior prevalência de comorbidades, principalmente hipertensão $(32,4 \%)$, diabetes $(17,6 \%)$ e dislipidemia $(8,8 \%)$. É importante destacar que os participantes com idade superior a 40 anos apresentaram taxa mais elevada de SM, estando presente em $61,8 \%$ nessa faixa-etária.

As variáveis que foram incluídas na análise multivariada e permaneceram no modelo final (valor-p<0,05), conforme apresentado na Tabela 4, foram: número de medicamentos e internação prévia (internação psiquiátrica). Observou-se que pacientes que tomam 4 medicamentos ou mais medicamentos por dia têm quase 7 vezes mais chance apresentar SM que aqueles que tomam até 3 medicamentos. Além disso, aqueles que nunca foram internados apresentam 8 vezes mais chance de apresentar SM que aqueles que já foram internados alguma vez. Destaca-se que o modelo apresentou bom ajuste de acordo com a estatística de Hosmer \& Lemeshow.

Tabela 1. Prevalência de síndrome metabólica e distribuição dos componentes utilizados para a classificação (n=72). Região Ampliada Oeste/Minas Gerais, 2015.

\begin{tabular}{lcc}
\hline & Frequência & Percentual (\%) \\
\hline Critérios & & \\
Pressão arterial elevada & 27 & 37,5 \\
Obesidade central & 32 & 44,4 \\
Hipertriglicemia & 46 & 63,9 \\
Baixo HDL & 15 & 20,8 \\
Hiperglicemia & 48 & 66,7 \\
Síndrome metabólica & & \\
Não & 38 & 52,8 \\
Sim & 34 & 47,2 \\
\hline
\end{tabular}


Tabela 2. Comparação das variáveis antropométricas e laboratoriais com a ocorrência de síndrome (n=72), Região Ampliada Oeste/Minas Gerais, 2015.

\begin{tabular}{|c|c|c|c|}
\hline \multirow{2}{*}{ Variáveis } & \multicolumn{2}{|c|}{ Síndrome Metabólica } & \multirow{2}{*}{ Valor-p } \\
\hline & Não $(n=38)$ & $\operatorname{Sim}(n=34)$ & \\
\hline \multicolumn{4}{|c|}{ Estado Nutricional } \\
\hline Eutrofia & $20(54,1 \%)$ & $5(14,7 \%)$ & $0,003^{*}$ \\
\hline Sobrepeso & $9(24,3 \%)$ & $16(47,1 \%)$ & \\
\hline Obesidade & $8(21,6 \%)$ & $13(38,2 \%)$ & \\
\hline \multicolumn{4}{|c|}{ Circunferência cintura } \\
\hline Normal & $30(78,9 \%)$ & $10(29,4 \%)$ & $<0,001^{\star}$ \\
\hline Alterado & $8(21,1 \%)$ & $24(70,6 \%)$ & \\
\hline \multicolumn{4}{|l|}{ PAS } \\
\hline Normal & $35(92,1 \%)$ & $21(61,8 \%)$ & $0,002^{\star}$ \\
\hline Alterado & $3(7,9 \%)$ & $13(38,2 \%)$ & \\
\hline \multicolumn{4}{|l|}{ PAD } \\
\hline Normal & $37(97,4 \%)$ & $28(82,4 \%)$ & $0,047 \dagger$ \\
\hline Alterado & $1(2,6 \%)$ & $6(17,6 \%)$ & \\
\hline \multicolumn{4}{|c|}{ Triglicérides } \\
\hline Normal & $22(57,9 \%)$ & $4(11,8 \%)$ & $<0,001^{\star}$ \\
\hline Alterado & $16(42,1 \%)$ & $30(88,2 \%)$ & \\
\hline \multicolumn{4}{|l|}{ HDL } \\
\hline Normal & $36(94,7 \%)$ & $21(61,8 \%)$ & $0,001 \dagger$ \\
\hline Alterado & $2(5,3 \%)$ & $13(38,2 \%)$ & \\
\hline \multicolumn{4}{|l|}{ Glicemia } \\
\hline Normal & $20(52,6 \%)$ & $5(14,7 \%)$ & $0,001^{\star}$ \\
\hline Alterado & $18(47,4 \%)$ & $29(85,3 \%)$ & \\
\hline
\end{tabular}

*Teste Qui-Quadrado de Pearson, †Teste exato de Fisher.

Tabela 3. Comparação das variáveis sociodemográficas, clínicas e comportamentais com a ocorrência de Síndrome Metabólica (n=72). Região Ampliada Oeste/Minas Gerais, 2015.

\begin{tabular}{llccc}
\hline Variáveis & & \multicolumn{2}{c}{ Síndrome Metabólica } & \multirow{2}{*}{ Valor-p } \\
\cline { 2 - 3 } & & Não $(\mathrm{n}=38)$ & Sim $(\mathrm{n}=34)$ & \\
\hline Sexo & Masculino & $57,90 \%$ & $41,20 \%$ & $0,157^{*}$ \\
\multirow{2}{*}{ Idade } & Feminino & $42,10 \%$ & $58,80 \%$ & \\
& Menos de 40 anos & $42,10 \%$ & $38,20 \%$ & $0,738^{*}$ \\
Estado civil & 40 anos ou mais & $57,90 \%$ & $61,80 \%$ & \\
& Sem parceiro & $84,20 \%$ & $70,60 \%$ & $0,165^{*}$ \\
Escolaridade & Com parceiro & $15,80 \%$ & $29,40 \%$ & \\
& Até fundamental completo & $51,40 \%$ & $63,60 \%$ & $0,300^{*}$ \\
Filhos & Ensino médio ou mais & $48,60 \%$ & $36,40 \%$ & \\
& Não & $75,70 \%$ & $61,80 \%$ & $0,205^{*}$ \\
& Sim & $24,30 \%$ & $38,20 \%$ & \\
\hline
\end{tabular}


Continuação Tabela 3.

\begin{tabular}{|c|c|c|c|c|}
\hline Trabalha atualmente & Sim & $5,30 \%$ & $14,70 \%$ & $0,243^{\star}$ \\
\hline & Não & $94,70 \%$ & $85,30 \%$ & \\
\hline Recebe aposentadoria & Sim & $64,70 \%$ & $71,90 \%$ & $0,532^{\star}$ \\
\hline & Não & $35,30 \%$ & $28,10 \%$ & \\
\hline Mora atualmente & Pais & $63,20 \%$ & $47,10 \%$ & $0,170^{*}$ \\
\hline & Outros & $36,80 \%$ & $52,90 \%$ & \\
\hline Renda familiar atual & Até 2 Salários Mínimos & $55,90 \%$ & $62,50 \%$ & $0,585^{\star}$ \\
\hline & 3 Salários Mínimos ou mais & $44,10 \%$ & $37,50 \%$ & \\
\hline Acompanhamento psiquiátrico & Rede Pública & $76,30 \%$ & $64,70 \%$ & $0,279^{*}$ \\
\hline & Rede particular & $23,70 \%$ & $35,30 \%$ & \\
\hline Tempo tratamento psiquiátrico & Até 10 anos & $29,40 \%$ & $34,40 \%$ & $0,665^{\star}$ \\
\hline & Acima de 10 anos & $70,60 \%$ & $65,60 \%$ & \\
\hline Internado em hospital psiquiátrico & Sim & $86,80 \%$ & $67,60 \%$ & $0,050^{*}$ \\
\hline & Não & $13,20 \%$ & $32,40 \%$ & \\
\hline Tempo usa clozapina & Até 5 anos & $24,20 \%$ & $35,50 \%$ & $0,325^{\star}$ \\
\hline & Acima de 5 anos & $75,80 \%$ & $64,50 \%$ & \\
\hline No de medicamentos & Até 3 medicamentos & $50,00 \%$ & $19,40 \%$ & $0,013^{\star}$ \\
\hline & 4 medicamentos ou mais & $50,00 \%$ & $80,60 \%$ & \\
\hline Comorbidades $\ddagger$ & Hipertensão & $7,90 \%$ & $32,40 \%$ & $0,052^{*}$ \\
\hline & Diabetes & $13,20 \%$ & $17,60 \%$ & \\
\hline & Dislipidemia & $0,00 \%$ & $8,80 \%$ & \\
\hline & Hipotireoidismo & $7,90 \%$ & $2,90 \%$ & \\
\hline & Psoríase & $2,60 \%$ & $0,00 \%$ & \\
\hline & Não possui & $73,70 \%$ & $58,80 \%$ & \\
\hline Fuma atualmente & Não & $78,90 \%$ & $70,60 \%$ & $0,413^{*}$ \\
\hline & Sim & $21,10 \%$ & $29,40 \%$ & \\
\hline Consome bebida alcoólica atualmente & Não & $2,70 \%$ & $2,90 \%$ & $0,999 \dagger$ \\
\hline & Sim & $97,30 \%$ & $97,10 \%$ & \\
\hline Pratica atividade física & Não & $73,00 \%$ & $51,60 \%$ & $0,069^{*}$ \\
\hline & Sim & $27,00 \%$ & $48,40 \%$ & \\
\hline
\end{tabular}

¥O mesmo paciente pode ter mais de uma doença.

*Teste qui-quadrado de Pearson, $†$ Teste exato de Fisher.

Tabela 4. Análise multivariada (regressão logística binária) avaliando os fatores associados à ocorrência de síndrome metabólica. Região Ampliada Oeste/Minas Gerais, 2015.

\begin{tabular}{|c|c|c|c|c|}
\hline \multirow{2}{*}{ Variavéis } & \multirow{2}{*}{ Valor-p } & \multirow{2}{*}{ OR } & \multicolumn{2}{|c|}{ IC 95\% para OR } \\
\hline & & & Limite inferior & Limite superior \\
\hline \multicolumn{5}{|l|}{ No de medicamentos } \\
\hline Até 3 medicamentos & - & 1 & - & - \\
\hline 4 medicamentos ou mais & 0,007 & 6,71 & 1,66 & 27,04 \\
\hline \multicolumn{5}{|l|}{ Foi internado } \\
\hline Sim & - & 1 & - & - \\
\hline Não & 0,014 & 8,04 & 1,53 & 42,17 \\
\hline
\end{tabular}

$\mathrm{OR}=$ Odds Ratio. IC=Intervalo Confiança. Valor-p Teste de Hosmer \& Lemeshow $=0,500$. 


\section{DISCUSSÃO E CONCLUSÃO}

$O$ resultado deste estudo mostra elevada prevalência de $\mathrm{SM}$ em pacientes que possuem esquizofrenia refratária na Região Ampliada de Saúde Oeste de Minas Gerais, evidenciando uma situação alarmante. Achado bastante similar foi encontrado em pesquisa realizada na Îndia, utilizando-se, também, dos critérios do NCEP ATP-III, na qual a prevalência de SM nesse grupo foi de $47 \%$, sendo essa taxa considerada elevada e, possivelmente, relacionada ao tratamento com clozapina e, também, às limitações impostas pela própria doença (20).

Poucos estudos, principalmente no Brasil e na América Latina, têm se dedicado a estabelecer e compreender a prevalência de SM em pessoas que possuem esquizofrenia refratária, sendo encontrada na literatura internacional uma variação de 46 a 69\% (12, 20, 21). Nebhinani (13) estimou a prevalência de SM em pessoas que fazem uso de clozapina antes e após 3 meses do início do tratamento, tendo apontado acréscimo de 33,3\% para $53,3 \%$, respectivamente, o que está próximo ao encontrado neste trabalho.

A razão para essa elevada prevalência ainda é muito controversa, principalmente em função da possível participação dos medicamentos antipsicóticos na gênese das anormalidades metabólicas. A esquizofrenia por si só já representa um fator de risco para as anormalidades que compõem a SM e, neste contexto, as medicações atípicas, entre elas a clozapina, possuem um papel importante na potencialização do risco (21). A relação precisa entre o uso de medicações antipsicóticas e o desenvolvimento de SM ainda permanece incerta, podendo-se afirmar com clareza que pessoas tratadas com esses medicamentos apresentam SM em uma frequência superior à da população geral. A prevalência de SM gira em torno de 4 a $26 \%$ nos pacientes que não fazem uso desses medicamentos, chegando a $69 \%$ naqueles medicados, nota- damente no grupo que possui esquizofrenia refratária (22).

Neste contexto, os antipsicóticos atípicos (segunda geração) representam um dos principais fatores de risco para o desenvolvimento de SM em pacientes que possuem esquizofrenia refratária (12). Quando comparados aos antipsicóticos típicos, estudos indicam que o uso de antipsicóticos atípicos aumenta de forma considerável o risco de alterações nos níveis de glicose, perfil lipídico e no peso corporal $(13,16,23)$. Os antipsicóticos atípicos podem ser classificados, atualmente, de acordo com a sua capacidade de induzir ganho de peso e diabetes. Nesse sentido, a clozapina é o fármaco com o maior potencial para SM dentre todos os existente e seu uso é associado ao acréscimo de três vezes no risco para SM, em comparação à classe de típicos (24). Uma recente investigação (25) mostrou que a clozapina está associada a taxas elevadas de SM, em comparação aos antipsicóticos típicos $(28,6$ e 70,4\%, p=0,004), o que indica a necessidade de priorização dos pacientes com esquizofrenia refratária no que diz respeito à monitorização clínica periódica e estratégias de promoção de hábitos saudáveis.

Mulheres apresentaram maior prevalência de SM em comparação aos homens, embora não tenha sido encontrada associação significativa. Um estudo que se propôs analisar as possíveis alterações metabólicas e genéticas em pacientes que faziam uso de clozapina mostrou que a prevalência no sexo feminino foi ligeiramente superior $(51,8 \%)$ (26). Em outra investigação, evidenciou-se que o sexo feminino apresentou maior risco para o desenvolvimento de SM em comparação ao masculino em pacientes com esquizofrenia refratária (25). Todavia percebe-se que os dados devem ser interpretados com parcimônia, tendo em vista os resultados discrepantes encontrados na literatura, o que indica a necessidade de prover os cuidados necessários a ambos os sexos (14). Além disso, sugere-se a construção de estudos longitudinais utilizando-se de randomização para elucidar melhor 
a participação do sexo na ocorrência de SM em pacientes com esquizofrenia refratária.

A idade também não se apresentou associada com a presença de SM nesta investigação. Entretanto, é importante destacar que a maior taxa de SM concentrou-se acima dos quarenta anos de idade. A prevalência de SM aumenta com a idade na população geral e, geralmente, verifica-se tendência semelhante na esquizofrenia, considerando-se que as maiores taxas são observadas na quarta e quinta décadas de vida $(27,28)$. Em pacientes que possuem esquizofrenia refratária verifica-se achado parecido, porquanto a prevalência, frequentemente, aumenta com a idade, com pico por volta dos quarenta anos $(26,29)$. Com base nesse contexto, sugere-se que o planejamento dos cuidados, tanto preventivos quanto relacionados ao tratamento, necessita priorizar pessoas com mais idade, sem, no entanto, deixar lado os mais jovens.

A prevalência de sobrepeso e de obesidade foi, consideravelmente, maior nos pacientes com a SM em comparação àqueles sem a síndrome. O índice de massa corporal (IMC) é, indiscutivelmente, o indicador antropométrico mais utilizado mundialmente, sendo extremamente útil na prática clínica para determinar a obesidade. Esse índice pode ser utilizado como uma medida de rastreamento de SM em pacientes que possuem esquizofrenia refratária e utilizam clozapina $(28,30)$. Estudo que comparou as alterações metabólicas possivelmente relacionadas ao uso de antipsicóticos mostrou que $52,3 \%$ dos indivíduos estavam com sobrepeso e indicou que aqueles que possuíam esquizofrenia refratária apresentaram as maiores taxas (31). Somando-se a isso, uma importante investigação retrospectiva corrobora que o aumento no IMC está diretamente associado à ocorrência de SM em pacientes com esquizofrenia refratária em uso de clozapina, indicando sua relevância como parâmetro a ser monitorado (32). Os achados desta investigação mostram que o IMC apresentou-se associado à ocor- rência de SM, alinhando-se ao demonstrado em outros estudos realizados com pacientes com esquizofrenia refratária que utilizavam clozapina $(33,34)$.

A análise dos componentes individuais da SM é importante para uma melhor compreensão do transtorno e de seus fatores de risco. Os pacientes com a síndrome apresentaram as maiores alterações em todos os parâmetros (pressão arterial, triglicérides, HDL-c, glicemia e CC), o que está em concordância com o estudo de Zhang et al. (26). Nesta investigação, os componentes que apresentaram as maiores prevalências foram a glicemia de jejum e triglicérides. Este resultado é ratificado por outro estudo semelhante realizado em Singapura (35). O uso de antipsicóticos atípicos, principalmente de clozapina, está relacionado ao aparecimento de complicações metabólicas, incluindo alteração no perfil lipídico $(31,36)$. A elevação dos níveis de triglicérides é um importante indicador de resistência à insulina e serve como um valioso marcador para avaliar pacientes com potencial risco para distúrbios metabólico, notadamente com esquizofrenia refratária (37).

A presença de comorbidades apresentouse associada à SM, sendo mais frequente nas pessoas acometidas com essa síndrome. Neste grupo, as mais prevalentes foram a hipertensão arterial sistêmica, diabetes mellitus do tipo II e dislipidemia. Tal achado pode ser comparado ao encontrado em uma recente investigação realizada com pacientes que possuíam esquizofrenia refratária, na qual a prevalência de hipertensão $(38,2 \%)$ e diabetes $(19,3 \%)$ assemelha-se ao deste estudo, porém sendo a dislipidemia a alteração mais prevalente $(48,7 \%)$ (15). A dislipidemia é uma das principais alterações encontradas em pacientes com esquizofrenia refratária, estando relacionada ao estilo de vida e ao uso de clozapina (38). As comorbidades são comuns em pacientes com esquizofrenia, aumentando o risco de desenvolvimento de SM 
e, por conseguinte, eventos cardiovasculares e morte $(31,37,39)$. Além disso, sabe-se que essas comorbidades aumentam significantemente o risco de morte prematura, chegando a ser três vezes maior do que o atribuído à população geral, principalmente no que se refere aos que possuem esquizofrenia refratária e utilizam clozapina (38). Pacientes que apresentam essas condições devem receber acompanhamento contínuo, além de serem incluídos em atividades e grupos de qualidade de vida e educação em saúde.

Pacientes com SM apresentaram um histórico de menos internações psiquiátricas, em comparação àqueles sem a SM, tendo este achado apresentado associação significativa incluído no modelo final. Sendo assim, infere-se que a presença de SM ou uma de suas complicações leve o paciente a uma maior procura pelos serviços de saúde, em função de suas questões clínicas, o que possivelmente pode contribuir, de forma concomitante, no controle mais adequado de seu quadro psiquiátrico e na identificação precoce de desestabilizações, diminuindo, por conseguinte, a necessidade de internação psiquiátrica. Entretanto, este resultado deve ser analisado com cautela, pois mais pesquisas devem ser realizadas, inclusive longitudinais, para qualquer generalização.

$\mathrm{O}$ uso de quatro ou mais medicamentos foi significativamente associado à ocorrência de SM, sendo observado que pacientes com a SM utilizam maior número de medicamentos que aqueles sem a síndrome. Uma pesquisa que verificou a relação entre SM e características clínicas em pacientes esquizofrênicos também identificou que aqueles que fizeram uso de dois ou mais medicamentos psiquiátricos apresentaram uma taxa de ocorrência da SM de 62,5\%, enquanto os que ingeriam apenas uma medicação apontaram uma taxa de 35,7\% (34).

A ingesta de vários medicamentos aumenta o risco de aparecimento de efeitos adversos metabólicos, contribuindo, por conse- guinte, para o desenvolvimento de SM (40). Esse achado deve ser visto com parcimônia, considerando-se que pacientes que possuem esquizofrenia refratária tendem a utilizar um número maior de medicamentos em detrimento da gravidade da doença e de sintomas diversos. Todavia, o uso de clozapina isoladamente é o principal preditor de risco para alterações metabólicas, independente da quantidade e classe de outros medicamentos utilizados, incluindo polifarmácia de antipsicóticos típicos ou primeira geração (25).

Verificou-se que a prevalência de SM em pessoas que possuem esquizofrenia refratária é alta, sendo este achado extremamente relevante. Sendo assim, indica-se que sejam planejadas e operacionalizadas ações que promovam a prevenção das alterações metabólicas e da obesidade, bem como estimulem o desenvolvimento de hábitos de vida saudáveis. Para isso, é necessário que esse grupo de pacientes seja visto como prioritário pelos gestores e profissionais de saúde, considerando-se a necessidade de vigilância contínua dessas alterações para o estabelecimento de intervenções precoces e efetivas. A promoção de estilo de vida saudável, com estímulo à prática de atividade física, pode diminuir o risco de SM e melhorar qualidade de vida desses pacientes (41).

As limitações deste estudo têm relação com sua validade externa, tendo em vista que a amostra não foi probabilística, o que implica necessidade de uma análise mais cautelosa no que tange à generalização dos resultados. O delineamento da pesquisa permitiu estimar a prevalência de SM, bem como os fatores associados, não sendo possível, em razão disso, realizar inferências de causa e efeito. Ademais, sugere-se o desenvolvimento de pesquisas longitudinais com o intuito de melhor elucidar a problemática da SM em pacientes com esquizofrenia refratária que fazem uso de clozapina. Todavia, este delineamento respondeu bem às questões norteadoras do estudo e aos objetivos da pesquisa. 


\section{REFERÊNCIAS}

1. Patel KR, Cherian J, Gohil K, Atkinson D. Schizophrenia: overview and treatment options. PT. 2014; 39(9): 638-45.

2. Brooke-Sumner C, Petersen I, Asher L, Mall S, Egbe CO, Lund C. Systematic review of feasibility and acceptability of psychosocial interventions for schizophrenia in low and middle income countries. BMC Psychiatry [Internet]. 2015 [citado 10 june 2015]; 15(19). Disponível em: http://www.biomedcentral.com/content/ pdf/s12888-015-0400-6.pdf.

3. Warnez S, Alessi-Severini S. Clozapine: a review of clinic practice guidelines and prescribing trends. BMC Psychiatry [Internet]. 2014 [citado 10 june 2015]; 14(102): 2-5. Disponível em: https:// www.ncbi.nlm.nih.gov/pmc/articles/ PMC3999500/

4. Mcllwain ME, Harrison J, Wheeler AJ, Russell BR. Pharmacotherapy for treatment-resistant schizophrenia. Neuropsychiatr Dis Treat. 2011; 7: 135-49.

5. Wang W, Pu C, Jiang J, Cao X, Wang J, Zhao $\mathrm{M}$, et al. Efficacy and safety of treating patients with refractory schizophrenia with antipsychotic medication and adjunctive electroconvulsive therapy: a systematic review and meta-analysis. Shanghai Arch Psychiatry. 2015; 27(4): 206-219.

6. Conley RR, Buchanan RW. Evaluation of treatment-resistant schizophrenia. Schizophr Bull. 1997; 23(4): 663-674.

7. Boyda HN, Procyshyn RM, Pang CC, Hawkes E, Wong D, Jin CH, et al. Metabolic side-effects of the novel second-generation antipsychotic drugs asenapine and iloperidone: a comparison with olanzapine. Plos One [Internet]. 2013 [citado 10 june 2015]; 8(1). Disponível em: http://journals.plos.org/plosone/article/ file?id=10.1371/journal.pone.0053459\&type $=$ printable

8. Burghardt KJ, Grove TB, Ellingrod VE.
Endothelial nitric oxide synthetase genetic variants, metabolic syndrome and endothelial function in schizophrenia. J Psychopharmacol. 2014; 28(4): 349-56.

9. Bonomini F, Rodella LF, Rezzani R. Metabolic syndrome, aging and involvement of oxidative stress. Aging Dis[Internet]. 2015 [citado 10 june 2015]; 6(2): 109-20. Disponível em: https://www.ncbi.nlm. nih.gov/pmc/articles/PMC4365955/

10. Mulé G, Calcaterra I, Nardi E, Cerasola $\mathrm{G}$, Cottone S. Metabolic syndrome in hypertensive patients: an unholy alliance. World J Cardiol [Internet]. 2014 [citado 10 june 2015]; 6(9): 890-907. Disponível em: https://www.ncbi.nlm.nih.gov/pmc/ articles/PMC4176799/

11. Beltrán-Sánchez H, Harhay MO, Harhay MM, McElligott S. Prevalence and trends of metabolic syndrome in the adult U.S. population, 1999-2010. J Am Coll Cardiol. 2013; 62(8): 697-703.

12. Papanastasiou E. The prevalence and mechanisms of metabolic syndrome in schizophrenia: a review. Ther Adv Psychopharmacol. 2013; 3(1): 33-51.

13. Nebhinani N, Grover S, Chakrabarti S, Kate N, Avasthi A. A longitudinal study of change in prevalence of metabolic syndrome and metabolic disturbances 3 months after Clozapine therapy.J Ment Health Human Behav. 2013; 18(1): 9-17.

14. Mitchell AJ. Is The prevalence of metabolic syndrome and metabolic abnormalities increased in early schizophrenia? A comparative meta-analysis of first episode, untreated and treated patients. Schizophr Bull. 2013; 39(2): 295-305.

15. Sicras-Mainar A, Maurino J, Ruiz-Beato E, Navarro-Artieda R. Prevalence of metabolic syndrome according to the presence of negative symptoms in patients with schizophrenia. Neuropsychiatr Dis Treat. 2015; 11: 51-7.

16. Zimbron J, Khandaker GM, Toschi C, Jones PB, Fernández-Egea E. A systematic review and meta-analysis of randomised 
controlled trials of treatments for clozapine-induced obesity and metabolic syndrome. Eur Neuropsychopharmacol. 2016; 26(9): 1353-1365.

17. Sociedade Brasileira de Cardiologia, Sociedade Brasileira de Hipertensão, Sociedade Brasileira de Nefrologia. VI diretrizes brasileiras de hipertensão arterial. Arq Bras Cardiol. 2010; 95(1Suppl 1): 1-51.

18. World Health Organization (WHO). Physical status: the use and interpretation of anthropometry [Internet]. Geneva: WHO; 1995 [citado 10 june 2015]. 452 p. Technical Report Series $N^{\circ} 854$. Disponível em: http://apps.who.int/iris/bitstream/10665/37003/1/WHO_TRS_854.pdf

19. Grundy SM, Cleeman JI, Daniels SR, Donato KA, Eckel RH, Franklin BA, et al. Diagnosis and management of the metabolic syndrome: an American Heart Association/National Heart, Lung, and Blood Institute Scientific Statement. Circulation. 2005; 112(17): 2735-52.

20. Grover S, Nebhinani N, Chakrabarti S, Avasthi A, Kulhara P. Metabolic syndrome among patients receiving clozapine: a preliminary estimate. Indian J Pharmacol. 2011; 43(5): 591-5.

21. Malhotra N, Grover S, Chakrabarti S, Kulhara P. Metabolic syndrome in schizophrenia. Indian J Psychol Med. 2013; 35(3): 227-40.

22. Burghardt KJ, Ellingrod VL. Detection of the metabolic syndrome in schizophrenia and implications for antipsychotic therapy: is there a role for folate? Mol Diagn Ther. 2013; 17(1): 21-30.

23. Vancampfort D, Stubbs B, Mitchell AJ, De Hert M, Wampers M, Ward PB, et al. Risk of metabolic syndrome and its components in people with schizophrenia and related psychotic disorders, bipolar disorder and major depressive disorder: a systematic review and meta-analysis. World Psychiatry. 2015 Oct; 14(3): 339-47.

24. Paredes RM, Quinones M, Marballi K, Gao X, Valdez C, Ahuja SS, et al. Me- tabolomic profiling of schizophrenia patients at risk for metabolic syndrome. Int J Neuropsychopharmacol. 2014; 17(8): 1139-48.

25. Softic R, Sutovic A, Avdibegovic E, Osmanović E, Bećirović E, Hajdukov MM. Metabolic syndrome in schizophrenia who is more to blame: fga polypharmacy or clozapine monotherapy? Psychiatr Danub. 2015; 27(4): 378-84.

26. Zhang Y, Chen M, Chen J, Wu Z, Yu S, Fang Y, et al. Metabolic syndrome in patients taking clozapine: prevalence and influence of catechol-O-methyltransferase genotype. Psychopharmacology (Berl). 2014; 231(10): 2211-8.

27. Hyde N, Dodd S, Venugopal K, Purdie C, Berk M, O’Neil A. Prevalence of cardiovascular and metabolic events in patients prescribed clozapine: a retrospective observational, clinical cohort study. Curr Drug Saf. 2015; 10(2): 125-31.

28. Sweileh WM, Zyoud SH, Dalal SA, Ibwini S, Sawalha AF, Ali I. Prevalence of metabolic syndrome among patients with Schizophrenia in Palestine. BMC Psychiatry. [Internet]. 2012 [citado 10 june 2015]; 12: 235-242. Disponível em: https:// www.ncbi.nlm.nih.gov/pmc/articles/ PMC3543707/pdf/1471-244X-12-235. pdf

29. Mitchell AJ, Vancampfort D, Sweers K, Van Winkel R, Yu W, De Hert M. Prevalence of metabolic syndrome and metabolic abnormalities in schizophrenia and related disorders: a systematic review and meta-analysis. Schizoph Bull. 2013; 39(2): 306-18.

30. Faasen N, Niehaus D, Koen L, Jordaan E. Undiagnosed metabolic syndrome and other adverse effects among clozapine users of Xhosa descent. S. Afr. j. psyc. 2014; 20(2): 54-57.

31. Francesco F, Cervone A. Metabolic alterations associated with first and second generation antipsychotics: an twenty-years open study. Psychiatr 
Danub. 2014; 26(Suppl 1): 184-7.

32. Bai YM, Lin CC, Chen JY, Chen TT, Su TP, Chou P Association of Weight Gain and Metabolic Syndrome in Patients Taking Clozapine: An 8-Year Cohort Study. J Clin Psychiatry. 2011; 72(6): 751-756.

33. Wysokiński A, Sobów T. Improvements in body composition, anthropometric measurements and lipid profile following discontinuation of clozapine. Nord J Psychiatry. 2016; 70(2): 156-60.

34. Saatcioglu O, Kalkan M, Fistikci N, Erek S, Kilic KC. Relationship between metabolic syndrome and clinical features, and its personal-social performance in patients with schizophrenia. Psychiatr Q [Internet]. 2015 [citado 10 june 2015]; 87(2): 265-80. Disponível em: http:// link.springer.com/article/10.1007\%2Fs11126-015-9384-0

35. Lee J, Nurjono M, Wong A, Salim A. Prevalence of metabolic syndrome among patients with schizophrenia in Singapore. Ann Acad Med Singapore. 2012; 41(10): 457-62.

36. Said MA, Hatim A, Habil MH, Zafidah W, Haslina MY, Badiah Y, et al. Metabolic syndrome and antipsychotic monotherapy treatment among schizophrenia patients in Malaysia. Prev Med. 2013; 57(Suppl): S50-3.

37. Gupta A, Dadheech G, Yadav D, Sharma $\mathrm{P}$, Gautam S. Metabolic Issues in schizophrenic patients receiving antipsychotic treatment. Ind J Clin Biochem. 2014; 29(2): 196-201.

38. Ventriglio A, Gentile A, Stella E, Bellomo A. Metabolic issues in patients affected by schizophrenia: clinical characteristics and medical management. Front Neurosci. 2015; 9: 297-303.

39. Gutiérrez-Rojas L, Azanza JR, Bernardo M, Rojo L, Mesa F, Martínez-Ortega JM. Prevalence of metabolic syndrome in Spanish patients with schizophrenia and overweight: the CRESSOB study. Actas Esp Psiquiatr. 2014; 42(1): 9-17.

40. Young SL, Taylor M, Lawrie SM. "First do no harm": a systematic review of the prevalence and management of antipsychotic adverse effects. J Psychopharmacol. 2015; 29(4): 353-62.

41. Eskelinen S, Sailas E, Joutsenniemi K, Holi M, Suvisaari J. Clozapine use and sedentary lifestyle as determinants of metabolic syndrome in outpatients with schizophrenia. Nord J Psychiatry. 2015; 69(5): 339-45. 